Research Article

\title{
Elucidating the Mechanisms of Hugan Buzure Granule in the Treatment of Liver Fibrosis via Network Pharmacology
}

\author{
Yi Zhu $\mathbb{D}^{1},{ }^{1}$ Ming Qiao $\mathbb{D}^{1},{ }^{1}$ Jianhua Yang $\mathbb{D},{ }^{1}$ and Junping $\mathrm{Hu} \mathbb{D}^{2}$ \\ ${ }^{1}$ Department of Pharmacy, The First Affiliated Hospital of Xinjiang Medical University, Urumqi 830000, China \\ ${ }^{2}$ College of Pharmacy, Xinjiang Medical University, Urumqi 830011, China0000 \\ Correspondence should be addressed to Junping Hu; hjp-yft@163.com
}

Received 4 August 2020; Revised 7 October 2020; Accepted 9 November 2020; Published 20 November 2020

Academic Editor: Jun Yan Tao

Copyright (c) 2020 Yi Zhu et al. This is an open access article distributed under the Creative Commons Attribution License, which permits unrestricted use, distribution, and reproduction in any medium, provided the original work is properly cited.

Objective. To holistically explore the latent active ingredients, targets, and related mechanisms of Hugan buzure granule (HBG) in the treatment of liver fibrosis (LF) via network pharmacology. Methods. First, we collected the ingredients of HBG by referring the TCMSP server and literature and filtered the active ingredients though the criteria of oral bioavailability $\geq 30 \%$ and drug-likeness index $\geq 0.18$. Second, herb-associated targets were predicted and screened based on the BATMAN-TCM and SwissTargetPrediction platforms. Candidate targets related to LF were collected from the GeneCards and OMIM databases. Furthermore, the overlapping target genes were used to construct the protein-protein interaction network and "drug-compoundtarget-disease" network. Third, GO and KEGG pathway analyses were carried out to illustrate the latent mechanisms of HBG in the treatment of LF. Finally, the combining activities of hub targets with active ingredients were further verified based on software AutoDock Vina. Results. A total of 25 active ingredients and 115 overlapping target genes of HBG and LF were collected. Besides, GO enrichment analysis exhibited that the overlapping target genes were involved in DNA-binding transcription activator activity, RNA polymerase II-specific, and oxidoreductase activity. Simultaneously, the key molecular mechanisms of HBG against LF were mainly involved in PI3K-AKT, MAPK, HIF-1, and NF- $\kappa$ B signaling pathways. Also, molecular docking simulation demonstrated that the key targets of HBG for antiliver fibrosis were IL6, CASP3, EGFR, VEGF, and MAPK. Conclusion. This work validated and predicted the underlying mechanisms of multicomponent and multitarget about HBG in treating LF and provided a scientific foundation for further research.

\section{Introduction}

Liver fibrosis (LF) is a pathophysiologic wound-healing response of the liver that is occasioned by chronic hepatic damages such as viral, alcoholic, and autoimmune hepatitis [1]. Epidemiological data have disclosed that LF gradually became the public focus that gives rise to extensive morbidity and mortality [2]. LF could develop into cirrhosis without favorable intervention, which is estimated to affect more than 1 million deaths per annum, and 55\% are caused by liver cirrhosis [3]. For the time being, a large number of evidence has proved that LF could be reversed with the appropriate intervention [4]. Consequently, it is extremely urgent to develop drugs with potential antiliver fibrosis activity.
Traditional Chinese Medicine (TCM) has gained wide attention in virtue of the conspicuous therapeutic effect and no obvious adverse reaction [5]. TCM also has the unique advantages of multicomponent, multitarget, and multipathway in the research field of antiliver fibrosis [6]. Therefore, further research on the material basis and the latent mechanisms of TCM is one of the ideas to develop TCM. Hugan buzure granule (HBG), a classical TCM formula, consists of Cuscuta chinensis, Chicory, Cumin, and Apium graveolens L.(Celery) and has been widely used in the clinical treatment of liver diseases [7]. Based on the previous research, HBG could diminish inflammatory cytokines and inhibit liver inflammatory damage and fibrosis, thereby preventing the liver from histopathological damages [8]. Due to the complex composition of HBG, it is hard to 
elucidate the latent mechanisms of HBG with multitarget and multipathway by means of the conventional assessment. So, this work aims to probe active compounds, targets, and synergistic mechanisms of HBG in the treatment of LF via network pharmacology (Figure 1).

\section{Materials and Methods}

2.1. Screening of Active Ingredients. The ingredients of the four herbs in $\mathrm{HBG}$ were acquired from a comprehensive retrieval of literature and the Traditional Chinese Medicine Systems Pharmacology database and analysis platform (TCMSP, http://lsp.nwu.edu.cn/tcmsp.php) [9]. Meanwhile, the parameters for screening the bioactive ingredients were set as follows: oral bioavailability $(\mathrm{OB}) \geq 30 \%$ and druglikeness (DL) $\geq 0.18$ [10]. Thus, the ingredients satisfying the abovementioned criteria were retained and regarded as candidate molecules of HBG for subsequent analysis. In addition, compounds with reported therapeutic effects on liver fibrosis were directly included.

2.2. Prediction and Screening of Potential Targets. The Bioinformatics Analysis Tool for Molecular mechanism of TCM (BATMAN-TCM, http://bionet.ncpsb.org/batman-tcm) and Swiss TargetPrediction (http://swisstargetprediction.ch/) databases were used to identify the bioactive ingredientassociated target genes of HBG together. The targets were further screened by the criteria of the "score cutoff $\geq 20$ and false discovery rate (FDR) $<0.05$ " in the BATMAN-TCM database [11]. Meanwhile, the predicted active ingredientassociated target genes in SwissTargetPrediction were filtered by presetting the boundary value of "gene probability $>0.7$ " [12]. Then, the protein names were transformed into the official symbols via the UniProt database (http://www. uniprot.org/) with the species limited to "Homo sapiens." LFassociated target genes were comprehensively retrieved through the Online Mendelian Inheritance in Man (OMIM, https://omim.org/) and GeneCards (https://www.genecards. org/) databases with "liver fibrosis" as the search term [13]. Finally, the potential targets of HBG against LF were identified and visualized by overlapping the abovementioned target genes with a Venn diagram (http:// bioinfogp.cnb.csic.es/tools/venny/index.html).

2.3. Network Construction. The Search Tool for the Retrieval of Interacting Genes/Proteins (STRING, https://string-db. org/) platform is a precomputed global resource, which could explore and build the interaction network of proteinprotein [14]. In this research, the overlapping targets were input into the STRING 11.0 with species simply limited to "Homo sapiens" and the interaction score $>0.40$ to implement the analysis of protein-protein interaction (PPI). Subsequently, the PPI network of targets intersection of HBG and LF was visualized via Cytoscape 3.8.0. The top 5 target proteins were acquired as the hub targets for HBG in the treatment of LF. The complex network relationship of "drug-compound-target-disease" was visualized via using Cytoscape 3.8.0. The nodes represented the compounds, targets, and disease, while the edges represented the interactions between them in the network. Besides, the degree represented the number of edges between nodes, which was analyzed via the Network Analyzer plugin of Cytoscape.

2.4. Gene Ontology (GO) and Signaling Pathway Analyses. GO (http://www.geneontology.org) and Kyoto Encyclopedia of Genes and Genomes (KEGG, http://www.kegg.jp/ pathway.html) signaling pathway analyses for overlapping targets were carried out using the Database for Annotation, Visualization, and Integrated Discovery (DAVID, https:// david.ncifcrf.gov/) with the "Homo sapiens" setting [15]. Additionally, GO can be applied to classify and elucidate the gene functions, which was performed according to the biological processes, cellular component, and molecular function [16]. The bubble chart of the concerned GO enrichment was plotted using the OmicShare tools. Moreover, the top 20 significant signaling pathways were screened in line with the ranking of gene count. In short, the mechanisms of HBG against LF were investigated by the biological functions and related signaling pathways of the overlapping targets. An FDR $<0.05$ in both GO and KEGG analyses was considered significant.

2.5. Molecular Docking. AutoDock Vina software can predict the binding activities of target proteins to compounds. The molecular docking of the top 5 hub targets and the active compounds was performed using AutoDock Vina software. The energy range, exhaustiveness, and num modes were, respectively, set as "10," "20," and “100." It is generally believed that the lower the binding energy, the more stably the active compound binds to the target.

\section{Results}

3.1. Active Compounds of $H B G$. Twenty-two active ingredients in $\mathrm{HBG}$ with $\mathrm{OB} \geq 30 \%$ and $\mathrm{DL} \geq 0.18$ were retrieved from the TCMSP server. Moreover, our previous studies indicated that dl-3n-butylphthalide, aesculetin, and apigenin were also regarded as active compounds in the treatment of LF [17]. In total, 25 active compounds were prepared for follow-up research, and the detailed information of them is shown in Table 1.

3.2. Target Genes Prediction. The predictive models containing BATMAN-TCM and SwissTargetPrediction platform were used to predict 132 target genes after duplicates elimination, which was associated with the 25 active ingredients of HBG. Moreover, 6376 target genes related to LF were retrieved from the GeneCards and OMIM databases. In brief, a total of 115 overlapping targets between active ingredients and LF after merging (Figure 2).

3.3. PPI Network. The PPI network was delineated via the STRING platform (Figure 3). In the PPI network, targets and relationship between targets were represented by nodes and edges. Connectivity degree, namely, is the number of lines 


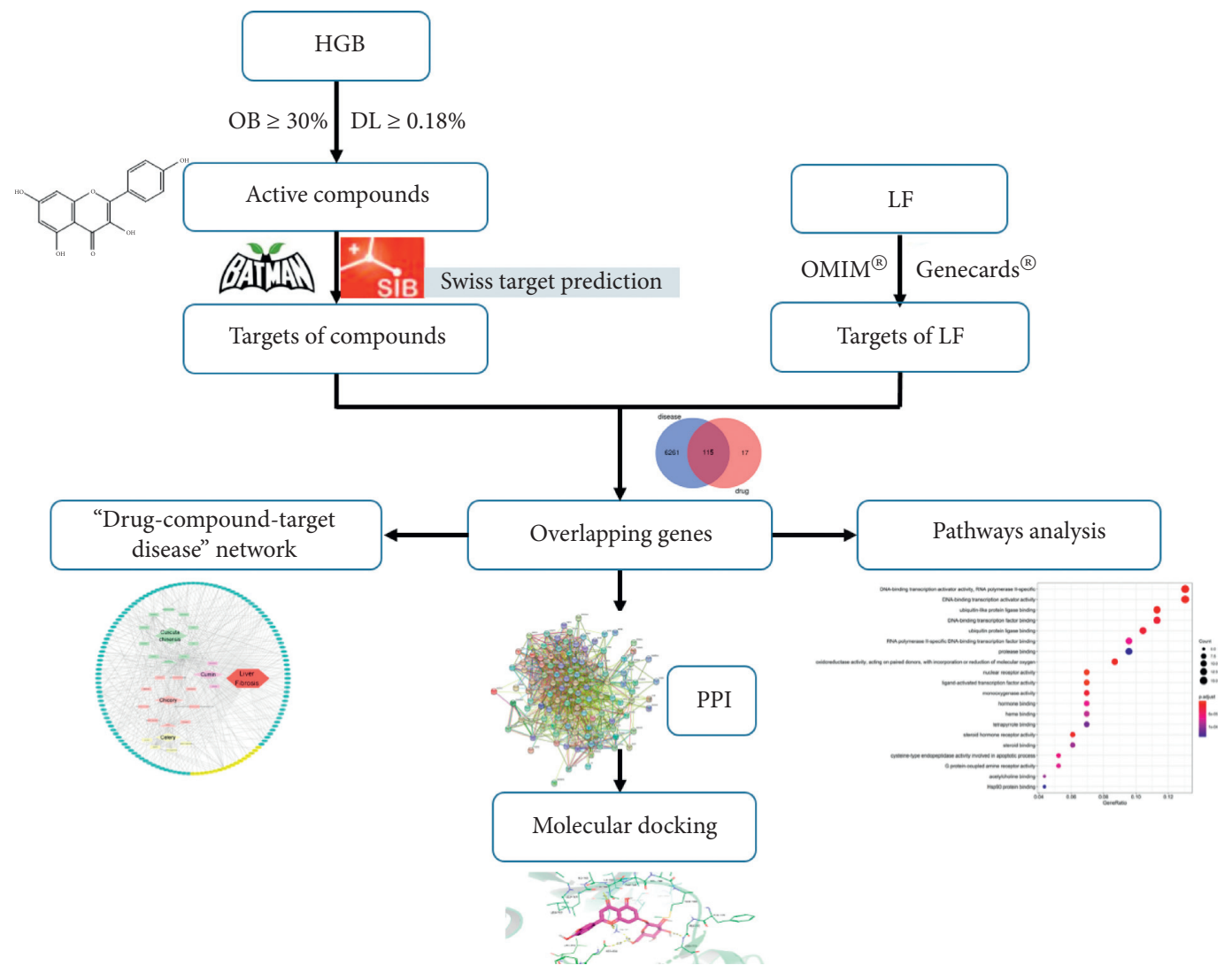

FIGURE 1: Flowchart of the network pharmacology of HBG in treating LF.

TABLE 1: Detailed information for 25 active compounds of HBG.

\begin{tabular}{|c|c|c|c|c|}
\hline Herb & Compound & OB $(\%)$ & $\mathrm{DL}$ & Degree \\
\hline \multirow{5}{*}{ Celery } & Dl-3n-butylphthalide & 47.9 & 0.07 & 41 \\
\hline & Aesculetin & 22.97 & 0.07 & 9 \\
\hline & Apigenin & 23.06 & 0.21 & 47 \\
\hline & Luteolin & 36.16 & 0.25 & 37 \\
\hline & Luteolin-7-o-glucoside & 7.29 & 0.78 & 23 \\
\hline \multirow{8}{*}{ Chicory } & Poriferast-5-en-3beta-ol & 36.91 & 0.75 & 12 \\
\hline & Delphinidin & 40.63 & 0.28 & 18 \\
\hline & Eseramine & 45.89 & 0.31 & 14 \\
\hline & 2S, 2'S-aurantiamide acetate & 39.18 & 0.54 & 34 \\
\hline & Lactucopicrin & 95.31 & 0.71 & 26 \\
\hline & (2R)-3-[3-(5-allyl-2-hydroxyphenyl)-4-hydroxyphenyl]propane-1, 2-diol & 32.21 & 0.20 & 18 \\
\hline & Gitoxigenin & 43.93 & 0.75 & 12 \\
\hline & Cyanidin 3-glucoside & 58.99 & 0.24 & 17 \\
\hline \multirow{2}{*}{ Cumin } & Ammidin & 34.55 & 0.22 & 18 \\
\hline & Stigmasterol & 43.83 & 0.76 & 31 \\
\hline \multirow{10}{*}{ Cuscuta chinensis } & Sesamin & 56.55 & 0.83 & 25 \\
\hline & NSC63551 & 39.25 & 0.76 & 23 \\
\hline & Isorhamnetin & 49.60 & 0.31 & 37 \\
\hline & Beta-sitosterol & 36.91 & 0.75 & 38 \\
\hline & Kaempferol & 41.88 & 0.21 & 40 \\
\hline & Campest-5-en-3beta-ol & 37.58 & 0.71 & 18 \\
\hline & Isofucosterol & 43.78 & 0.76 & 12 \\
\hline & Matrine & 63.77 & 0.25 & 11 \\
\hline & CLR & 37.87 & 0.68 & 24 \\
\hline & Quercetin & 46.43 & 0.28 & 31 \\
\hline
\end{tabular}




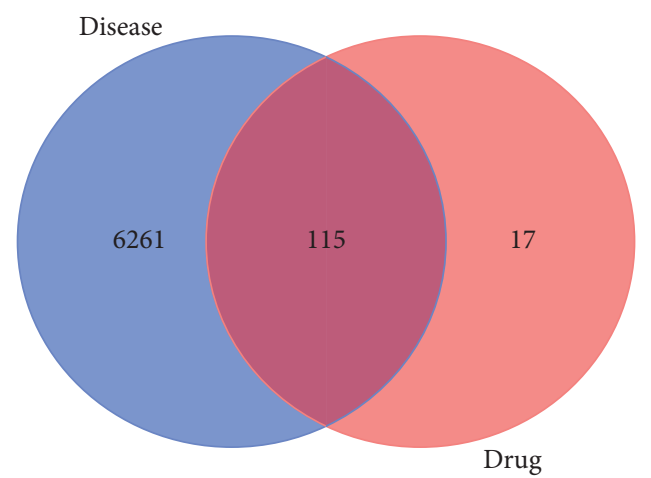

FIGURE 2: Venn diagram of the target genes for active ingredients and LF. Active ingredients have 132 target genes, while LF has 6376 target genes. There are 115 overlapping target genes between the two sets.

linked to a certain node. Accordingly, a target with a high connectivity degree in the PPI network might be a crucial target for the disease. The PPI network contained 114 nodes and 1160 edges. In addition, the top 5 targets were IL6 (67), CASP3 (63), EGFR (62), VEGF (61), and MAPK (60), respectively. All of these manifested the top targets coordinatively to treat LF at the molecular level. For instance, interleukin-6 (IL6) is one of the most important inflammatory cytokines. The IL6 signal pathway is involved in the regulation of profibrogenic and proinflammatory responses [18]. Additionally, the vascular endothelial growth factor (VEGF) is related the progression of LF via affecting the function of hepatic stellate cells (HSCs) [19]. Mitogenactivated protein kinase (MAPK) plays a key role in many processes of physiological and pathological such as inflammation, apoptosis, cell cycle, and growth [20]. Additionally, caspase-3 (CASP3) is activated to trigger the irreversible apoptosis [21].

3.4. "Drug-Compound-Target-Disease" Network. This work constructed a "drug-compound-target-disease" interaction network to visualize the relationship between drugs, active components, targets, and disease, and the interactions among them are represented in Figure 4. Generally, the degree of target interactions is an indicator of the potential significance of the compounds. In this research, apigenin $($ degree $=47), \quad$ dl-3n-butylphthalide $\quad($ degree $=41), \quad$ and kaempferol (degree $=40)$ with a high connectivity degree might be the core compounds in the pharmacological effect of HBG (Table 1). The "drug-compound-target-disease" network demonstrated that multiple targets were associated with various ingredients in different herbs, which might exhibit synergistic effects or additive effects of HBG in the treating LF. It was consistent with the common characteristics of TCM, in which multicomponent and multitarget were observed.

3.5. DAVID Pathway Analysis. GO and signaling pathway analyses were performed in order to clarify the underlying mechanisms of HBG on LF. In this research, $134 \mathrm{GO}$ terms and 127 signaling pathways were obtained (FDR $<0.05)$. The top 20 terms of GO enrichment were illustrated in Figure 5, including DNA-binding transcription activator activity, RNA polymerase II-specific, ubiquitin-like protein ligase binding, and oxidoreductase activity. The signaling pathways of $\mathrm{HBG}$ in treating LF were mostly involved in the PI3K-AKT signaling pathway, MAPK signaling pathway, HIF-1 signaling pathway, and NF- $\kappa \mathrm{B}$ signaling pathway(Table 2).

3.6. Molecular Docking. The top 5 hub targets in the PPI network were docked with the active ingredients (Table 3, Figure 6). The binding energy (affinity) was selected as the group representative. It was usually considered that the value of binding energy represented the binding activity between a compound and a certain protein, and the lower the binding energy, the more stably the active compound binds to the target. The results showed that the binding ability between EGFR and luteolin-7-o-glucoside was the best in all, followed by MAPK and sesamin, CASP3, IL6, and luteolin-7-o-glucoside, and VEGF and delphinidin. In total, the molecular docking revealed that the active compounds of HBG had good binding activities to key targets. The dotted yellow line in Figure 6 represents hydrogen bonds.

\section{Discussion}

LF is regarded as a crucial pathological procedure in the development of cirrhosis or hepatocellular carcinoma, with complicated formation procedures and molecular mechanisms, so the effective treatment of LF still confronts great challenges [22]. Although the preceding studies have carried out primary research on $\mathrm{HBG}$, the scientific material basis and molecular mechanisms in the treatment of LF have still not been systematically illuminated. Hence, a new analytical method is needed which could link the HBG to its target genes with the purpose of proving its relationships with the observed biological efficacy. Due to the intricate compounds in $\mathrm{HBG}$ and the diverse target genes in vivo, this work explored the material basis and mechanisms of HBG via network pharmacology. The systematic and holistic characteristic of network pharmacology is identical with the integral view of TCM [23]. It can illustrate the underlying mechanisms of $\mathrm{HBG}$ in treating $\mathrm{LF}$ via using existing 


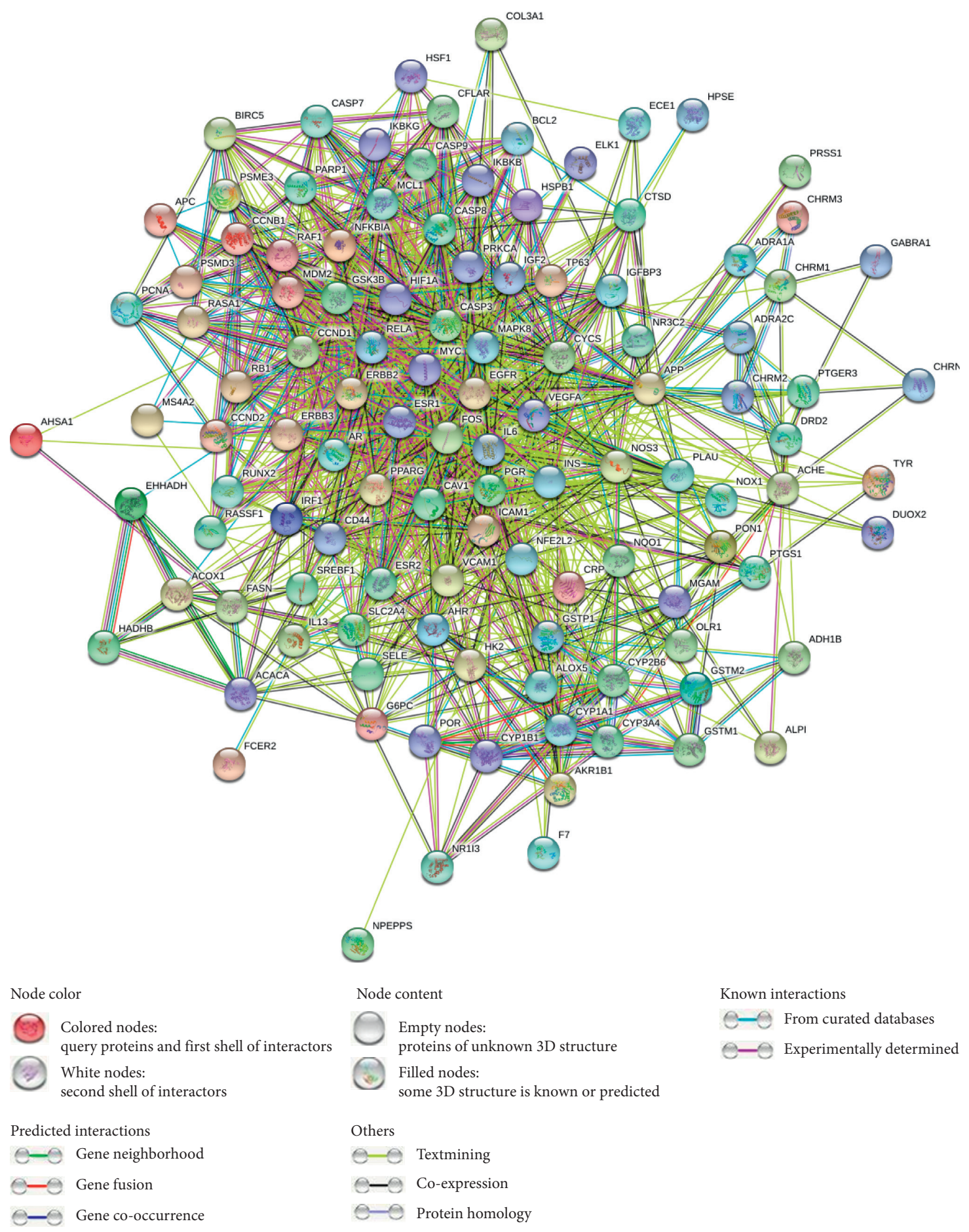

Figure 3: PPI network of targets intersection of LF and HBG.

databases and network analysis platforms. In the present study, 25 bioactive ingredients, 115 latent targets, and 127 related signaling pathways in HBG were predicted by network pharmacological analysis. Among them, IL6, CASP3, EGFR, VEGF, and MAPK were hub target genes of HGB in treating LF. Simultaneously, the key molecular mechanisms of HBG against LF were mainly involved in PI3K-AKT, MAPK, HIF-1, and NF- $\kappa$ B signaling pathways.

This research manifested that apigenin, 3-nbutylphthalide, and kaempferol might be the pivotal compounds in the pharmacological effect of HBG. Our previous research has indicated that apigenin could mitigate liver inflammation and fibrosis in rats by MAPK, PI3K/AKT, HIF-1, and eNOS signaling pathways [24]. Moreover, the preceding study has revealed that 3-n-butylphthalide, aesculetin, and quercetin could decrease the synthesis of the hepatic extracellular matrix (ECM) to alleviate LF via regulating proliferation and apoptosis of hepatic HSCs [17]. Besides, it has been reported that kaempferol might restrain the proliferation of liver cancer HepG2 cells by deactivating 


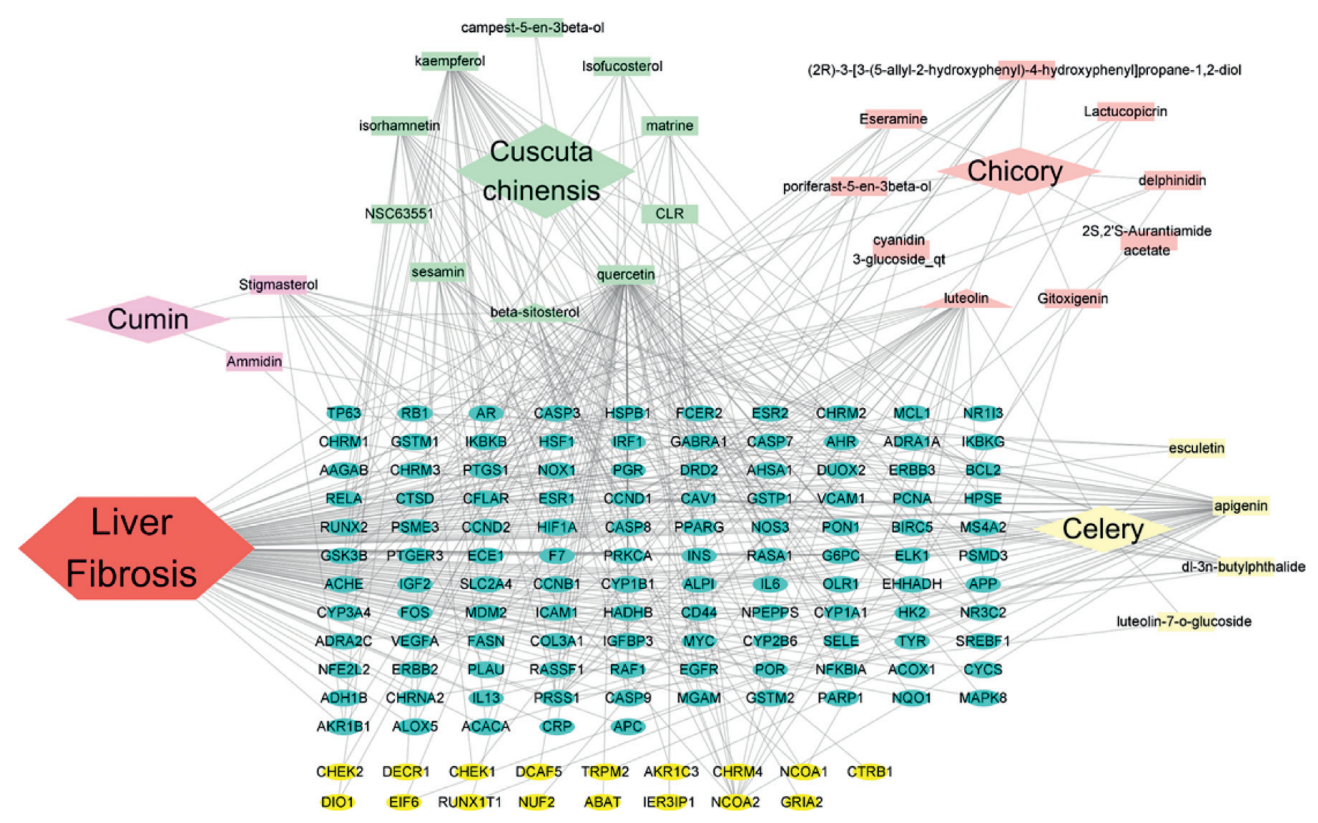

FIgURE 4: Network of the drug, active ingredients, targets, and LF. Red hexagon, LF; diamond, drug; rectangle, active compounds; and oval, targets.

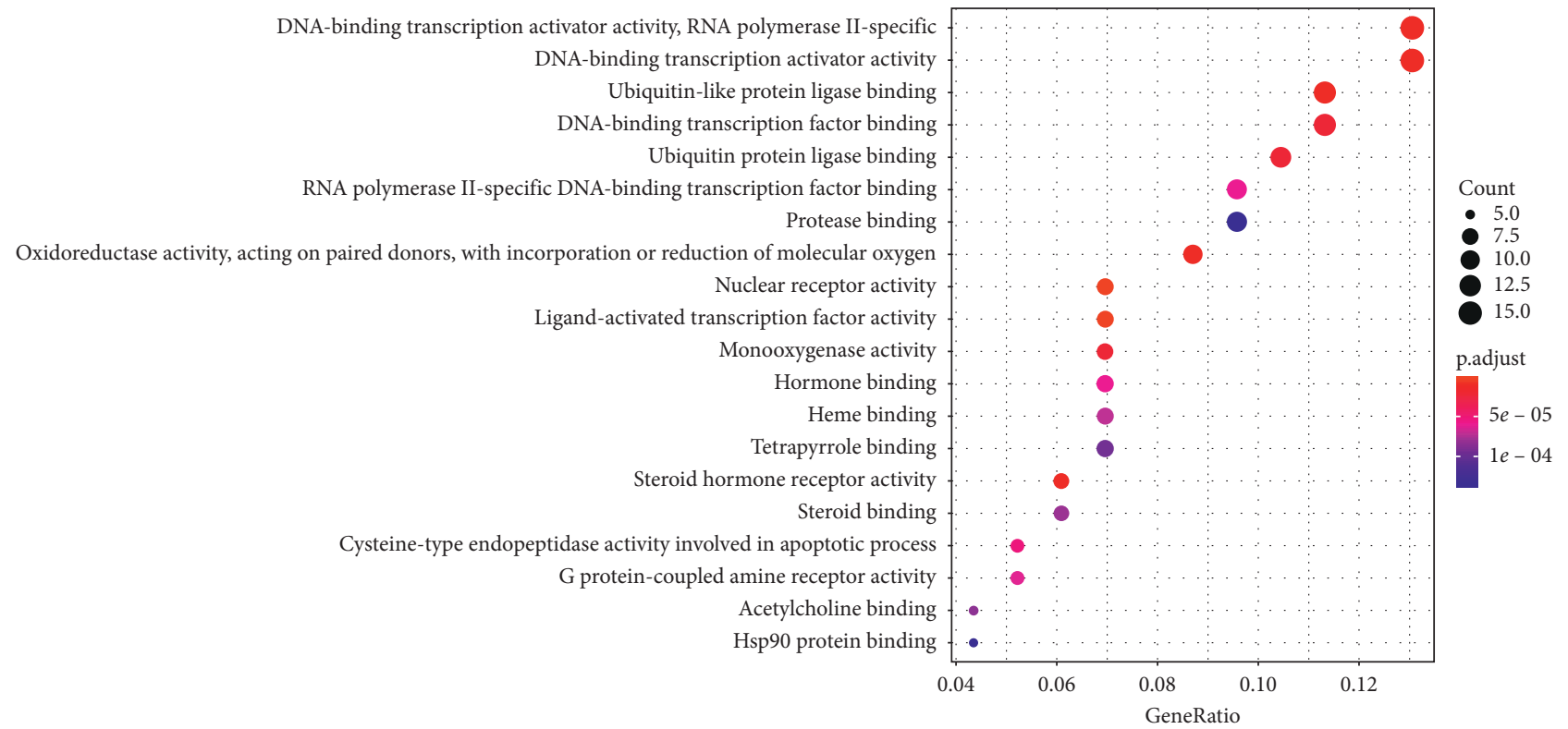

FIGURE 5: The bubble chart for GO analysis of the overlapping targets.

the PI3K/AKT signaling pathway [25]. In conclusion, multiple components of HBG might synergistically ameliorate LF.

Hub targets are closely interconnected with nodes in a PPI network, which proved that they have highly correlation in a certain disease. Simultaneously, IL6, CASP3, EGFR, VEGF, and MAPK were predicted as hub targets which possessed high connection degree in the PPI network. It suggested that HBG has the characteristics of multicomponent and multitarget in treating LF. IL6 is a profibrogenic and proinflammatory cytokine which can accelerate the occurrence of LF via increasing the deposition of ECM [26]. Several studies have demonstrated the rise of IL6 with the exacerbation of LF, which has a positive correlation with the ECM levels of hyaluronic acid, laminin, type IV collagen, and phase of LF, manifesting that the raise of the IL6 level could give rise to the development of LF [27]. The activation of CASP3 is regarded as the irreversible stage of apoptosis. Meanwhile, it has been found that the drug can mitigate LF by antagonizing the caspase receptor or suppressing the expression of key proteins in caspase, thereby preventing the cascade reaction of hepatocyte apoptosis [28]. Furthermore, 
TABLE 2: KEGG analysis of the overlapping targets (top 20).

\begin{tabular}{|c|c|c|c|}
\hline Name of pathways & Count & FDR & Enriched genes \\
\hline $\begin{array}{l}\text { PI3K-AKT signaling } \\
\text { pathway }\end{array}$ & 24 & $4.47 E-10$ & $\begin{array}{c}\text { BCL2, CHRM1, VEGFA, MYC, GSK3B, MDM2, EGFR, ERBB2, G6PC, MCL1, IL6, ERBB3, } \\
\text { RELA, NOS3, IKBKG, CCND1, INS, IKBKB, CASP9, RAF1, IGF2, CHRM2, CCND2, } \\
\text { PRKCA }\end{array}$ \\
\hline $\begin{array}{l}\text { MAPK signaling } \\
\text { pathway }\end{array}$ & 18 & $6.39 E-10$ & $\begin{array}{c}\text { VEGFA, MYC, EGFR, ERBB2, RASA1, CASP3, ELK1, ERBB3, FOS, RELA, HSPB1, IKBKG, } \\
\text { INS, IKBKB, MAPK8, RAF1, IGF2, PRKCA }\end{array}$ \\
\hline HIF-1 signaling pathway & 15 & $3.06 E-09$ & $\begin{array}{l}\text { BCL2, VEGFA, EGFR, ERBB2, IL6, RELA, HIF1A, NOS3, INS, HK2, PRKCA, CFLAR, } \\
\text { CASP8, ICAM1, VCAM1 }\end{array}$ \\
\hline $\begin{array}{l}\text { NF- } \kappa \text { B signaling } \\
\text { pathway }\end{array}$ & 11 & $3.09 E-09$ & BCL2, NFKBIA, RELA, IKBKG, IKBKB, CFLAR, PLAU, PARP1, ICAM1, VCAM1, EGFR \\
\hline Hepatitis B & 10 & $5.34 E-09$ & BCL2, MYC, NFKBIA, IL6, CASP3, ELK1, FOS, RELA, CYCS, BIRC5 \\
\hline Hepatitis C & 10 & $4.98 E-08$ & MYC, GSK3B, EGFR, NFKBIA, CASP3, RELA, CYCS, IKBKG, CCND1, MAPK8 \\
\hline TNF signaling pathway & 9 & $5.95 E-08$ & NFKBIA, IRF1, SELE, CASP7, IL6, CASP3, FOS, RELA, RB1 \\
\hline $\begin{array}{l}\text { Hepatocellular } \\
\text { carcinoma }\end{array}$ & 9 & $3.38 E-07$ & GSTP1, NFE2L2, MYC, GSK3B, EGFR, ELK1, NQO1, CCND1, GSTM1 \\
\hline Ras signaling pathway & 8 & $4.74 E-07$ & VEGFA, EGFR, RASA1, ELK1, RELA, RASSF1, IKBKG, INS \\
\hline FoxO signaling pathway & 7 & $5.18 E-07$ & CCNB1, MDM2, EGFR, G6PC, IL6, SLC2A4, CCND1 \\
\hline $\begin{array}{l}\text { Insulin signaling } \\
\text { pathway }\end{array}$ & 7 & $1.19 E-06$ & INS, IKBKB, MAPK8, SREBF1, FASN, RAF1, HK2 \\
\hline Focal adhesion & 7 & $1.42 E-06$ & BCL2, VEGFA, GSK3B, EGFR, ERBB2, ELK1, CAV1 \\
\hline Pancreatic cancer & 6 & $1.76 E-06$ & VEGFA, EGFR, ERBB2, RELA, IKBKG, CCND1 \\
\hline IL-17 signaling pathway & 6 & $2.50 E-06$ & GSK3B, NFKBIA, IL6, CASP3, FOS, RELA \\
\hline $\begin{array}{l}\text { VEGF signaling } \\
\text { pathway }\end{array}$ & 6 & $5.57 E-06$ & VEGFA, NOS3, HSPB1, CASP9, RAF1, PRKCA \\
\hline p53 signaling pathway & 5 & $9.47 E-06$ & CCNB1, BCL2, MDM2, IGFBP3, CASP3 \\
\hline $\begin{array}{l}\text { Relaxin signaling } \\
\text { pathway }\end{array}$ & 5 & $2.26 E-05$ & VEGFA, EGFR, NFKBIA, COL3A1, FOS \\
\hline $\begin{array}{l}\text { Calcium signaling } \\
\text { pathway }\end{array}$ & 5 & $3.86 E-05$ & CHRM1, ADRA1A, EGFR, ERBB2, CHRM3 \\
\hline $\begin{array}{l}\text { cAMP signaling } \\
\text { pathway }\end{array}$ & 4 & $6.05 E-05$ & FOS, RELA, MAPK8, ACOX1 \\
\hline $\begin{array}{l}\text { AMPK signaling } \\
\text { pathway }\end{array}$ & 4 & $9.76 E-05$ & INS, SREBF1, FASN, PPARG \\
\hline
\end{tabular}

studies have shown that the downstream signal pathways of the epidermal growth factor receptor (EGFR) were activated in HSCs when the liver was damaged and the phosphorylation levels of AKT, Smad, and Cyclin D1 were upregulated, promoting the increase of ECM and accelerating the generation of LF [29]. Additionally, the VEGF plays a vital role in the pathological progress of fibrogenesis. Recent publications have indicated that the level of serum VEGF was increased via activated $\mathrm{HSC}$ after $\mathrm{CCl}_{4}$ intervention, which was related to the development of LF $[30,31]$. MAPK is an indispensable protein that keeps the balance between antiinflammatory and proinflammatory responses. Studies have reported that suppression of MAPK alleviated LF by repression of inflammatory factors [32]. These findings indicated the potential antifibrosis activity of hub targets of HBG in LF.

According to the KEGG metabolic pathway analysis, the PI3K-AKT, MAPK, HIF-1, and NF- $\kappa$ B signaling pathways were mainly involved in treating LF with HBG. With the study of a great deal of literature, the phosphatidylinositol 3 kinase/protein kinase B (PI3K/AKT) signaling is closely linked to the activation, proliferation, and ECM deposition of HSCs [33, 34]. Simultaneously, acceleration of ECM deposition is related to all sorts of fibrosis diseases via the stimulated PI3K/AKT pathway. Conversely, blocking of the
PI3K/AKT pathway in HSCs can decrease fibrosis factors, collagen synthesis, and ECM deposition [35]. The mitogenactivated protein kinase family (MAPKs) cascade primarily containing p38 MAPK, JNK, and ERK can regulate elementary cellular signals including proliferation, apoptosis, differentiation, and inflammation [36, 37]. The activation of p38 MAPK, JNK, and ERK could accelerate the release of proinflammatory factors which further exacerbate LF $[38,39]$. Meanwhile, several studies have manifested that interruption of the MAPK pathway alleviated LF via diminishing the expression of inflammatory factors [40]. Hypoxia is inevitable in the local microenvironment during liver injury and secondary inflammatory reaction [41]. Hypoxiainducible factor 1 (HIF-1) consisted of oxygenregulated HIF- $1 \alpha$ subunit and HIF- $1 \beta$ subunit, which activates a great many of hypoxia responsive factors to adapt to the hypoxic microenvironment [42]. Another study reported that HIF- $1 \alpha$ might ameliorate LF via regulating expression of genes for angiogenesis and collagen synthesis [43]. Nuclear transcription factor-kappa $\mathrm{B}(\mathrm{NF}-\kappa \mathrm{B})$ is a pivotal transcription factor, which could govern inflammatory response by taking part in the activation procedure of inflammatory and macrophages cells $[44,45]$. Interestingly, it has been well reported that suppression of the NF- $\kappa \mathrm{B}$ pathway and changed regulation of $\mathrm{NF}-\kappa \mathrm{B}$-dependent gene transcription 
TABLE 3: Docking results of the top 5 hub targets with active compounds.

\begin{tabular}{|c|c|c|c|c|c|c|}
\hline \multirow{2}{*}{ No. } & \multirow{2}{*}{ Compound } & \multicolumn{5}{|c|}{ Affinity $\left(\mathrm{kJ} \cdot \mathrm{mol}^{-1}\right)$} \\
\hline & & IL6 & CASP3 & EGFR & VEGF & MAPK \\
\hline 1 & Dl-3n-butylphthalide & -5.4 & -5.2 & -6.4 & -5.3 & -8.5 \\
\hline 2 & Esculetin & -6.0 & -5.1 & -7.2 & -5.3 & -6.7 \\
\hline 3 & Apigenin & -6.4 & -6.4 & -8.2 & -6.8 & -9.0 \\
\hline 4 & Luteolin & -6.6 & -6.8 & -8.4 & -7.1 & -8.9 \\
\hline 5 & Luteolin-7-o-glucoside & -7.5 & -7.6 & -10.0 & -7.2 & -9.2 \\
\hline 6 & Poriferast-5-en-3beta-ol & -5.5 & -6.7 & -8.2 & -5.7 & -7.7 \\
\hline 7 & Beta-sitosterol & -5.7 & -6.4 & -9.1 & -5.8 & -7.7 \\
\hline 8 & Delphinidin & -6.6 & -6.6 & -8.7 & -7.4 & -8.5 \\
\hline 9 & Eseramine & -7.1 & -6.5 & -7.6 & -6.1 & -8.1 \\
\hline 10 & 2S, 2'S-aurantiamide acetate & -6.6 & -7.0 & -9.0 & -6.2 & -7.8 \\
\hline 11 & Lactucopicrin & -6.0 & -8.0 & -9.2 & -6.3 & -7.9 \\
\hline 12 & (2R)-3-[3-(5-allyl-2-hydroxyphenyl)-4-hydroxyphenyl]propane-1, 2-diol & -6.2 & -6.6 & -8.0 & -6.0 & -8.1 \\
\hline 13 & Gitoxigenin & -6.2 & -6.8 & -8.5 & -6.0 & -7.2 \\
\hline 14 & Cyanidin 3-glucoside & -6.4 & -6.1 & -8.5 & -7.3 & -8.6 \\
\hline 15 & Ammidin & -6.0 & -6.1 & -8.2 & -6.7 & -8.0 \\
\hline 16 & Stigmasterol & -6.2 & -6.6 & -8.5 & -6.9 & -8.0 \\
\hline 17 & Sesamin & -7.2 & -7.3 & -9.7 & -7.1 & -9.5 \\
\hline 18 & NSC63551 & -6.4 & -7.2 & -9.3 & -5.9 & -8.3 \\
\hline 19 & Isorhamnetin & -6.5 & -6.7 & -8.3 & -6.8 & -8.8 \\
\hline 20 & Kaempferol & -6.4 & -6.4 & -8.0 & -6.7 & -8.8 \\
\hline 21 & Campest-5-en-3beta-ol & -6.1 & -6.1 & -9.1 & -5.5 & -7.5 \\
\hline 22 & Isofucosterol & -5.8 & -6.5 & -9.5 & -5.8 & -7.9 \\
\hline 23 & Matrine & -5.7 & -6.0 & -6.7 & -5.5 & -7.4 \\
\hline 24 & CLR & -5.3 & -6.3 & -7.8 & -5.8 & -7.3 \\
\hline 25 & Quercetin & -6.5 & -6.8 & -8.5 & -7.2 & -8.5 \\
\hline
\end{tabular}

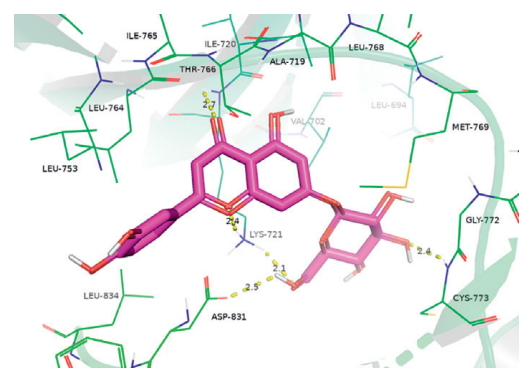

(a)

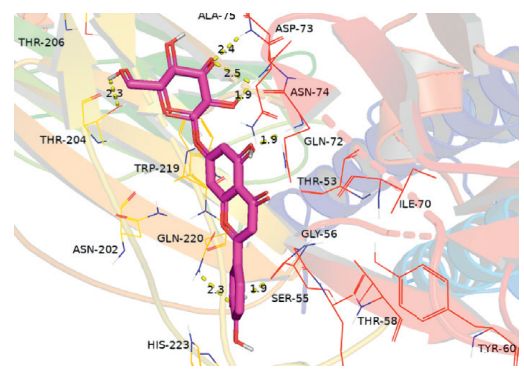

(d)

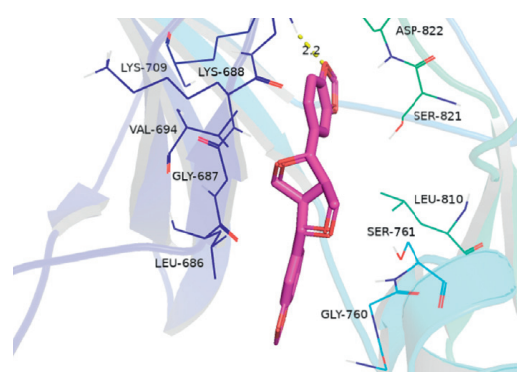

(b)

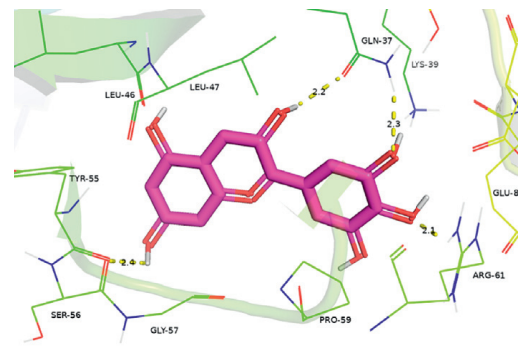

(e)

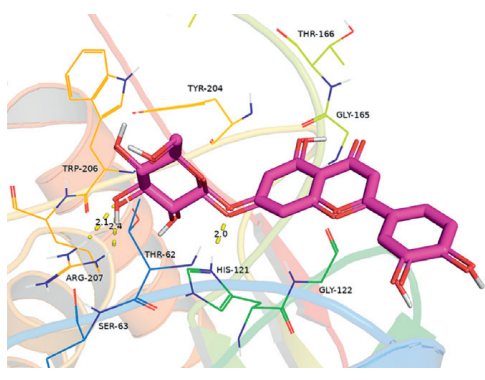

(c)

FIGURE 6: The molecular docking of the compound target. (a) EGFR and luteolin-7-o-glucoside. (b) MAPK and sesamin. (c) CASP3 and luteolin-7-o-glucoside. (d) IL6 and luteolin-7-o-glucoside. (e) VEGF and delphinidin.

can induce HSCs apoptosis which may further mitigate the severity of LF [46]. HBG also mitigated LF through PDGF, TNF, VEGF, and IL-17 signaling pathways in addition to the abovementioned main signaling pathways. The plateletderived growth factor (PDGF) is a ubiquitous cytokine that promotes cell activation, division, and proliferation and plays an important role in the development of LF [47]. The tumor necrosis factor (TNF) pathway is associated with cell apoptosis, survival, and inflammation [48]. The VEGF, the most potent angiogenesis cytokine, plays a crucial role in the 
pathological process of fibrogenesis [49]. As a characteristic cytokine, Interleukin-17 (IL-17) regulates the local or systemic inflammatory response [50]. In summary, it was further proved that multicomponent and multitarget of HBG could participate cooperatively in regulating multiple metabolic pathways, such as PI3K-AKT, MAPK, HIF-1, NF$\kappa \mathrm{B}$, PDGF, TNF, VEGF, and IL-17 signaling pathways, thereby exerting anti-inflammatory and antifibrotic effects.

Several limitations existed in the prediction of active ingredients and underlying mechanisms for HBG via network pharmacology. First, network pharmacology cannot reflect the influence of herb compatibility interaction in prescription. Second, the screening of $\mathrm{OB}$ and DL of compounds also had limitations, only preserved the information of TCMSP, yet had no regard for other bioactive compounds in treating LF. Third, the active components selected were not necessarily the drug components actually absorbed into the body after oral administration. Consequently, it requires to be verified in follow-up pharmacological experiments to further development of HBG.

\section{Conclusions}

This work firstly explored the active components, potential targets, and mechanisms of HBG against LF based on a systematical perspective by network pharmacologymolecular docking. In this work, 25 active ingredients, 115 crucial targets, and 127 related signaling pathways in HBG were predicted. Among them, IL6, CASP3, EGFR, VEGF, and MAPK were hub targets of HGB in treating LF. Simultaneously, the key molecular mechanisms of HBG against LF were mainly involved in PI3K-AKT, MAPK, HIF1 , and NF- $\kappa \mathrm{B}$ signaling pathways. It unfolded the characteristics of multicomponent, multitarget, and multipathway in HBG for the treatment of LF. In brief, this work broadens the line of thought for further pharmacological research.

\section{Data Availability}

The data used to support the findings of this study are available from the authors.

\section{Conflicts of Interest}

The authors declare that there are no conflicts of interest regarding the publication of this article.

\section{Authors' Contributions}

Yi Zhu and Ming Qiao contributed equally to this work.

\section{Acknowledgments}

This research was supported by Xinjiang Key Laboratory of Natural Active Components and Drug Release Technology (XJDX1713), the National Natural Science Foundation of China (No.81560688), and Special Funds for the Construction of Key Disciplines during the 13th Five-Year Plan in Xinjiang Uygur Autonomous Region.

\section{References}

[1] Y. Zhou, X. Lv, H. Qu et al., "Differential expression of circular RNAs in hepatic tissue in a model of liver fibrosis and functional analysis of their target genes," Hepatology Research, vol. 49, no. 3, pp. 324-334, 2019.

[2] D. Huang, T. Lin, S. Wang et al., "The liver fibrosis index is superior to the APRI and FIB-4 for predicting liver fibrosis in chronic hepatitis B patients in China," BMC Infectious Diseases, vol. 19, no. 1, pp. 878-891, 2019.

[3] H.-C. Tsay, Q. Yuan, A. Balakrishnan et al., "Hepatocytespecific suppression of microRNA-221-3p mitigates liver fibrosis," Journal of Hepatology, vol. 70, no. 4, pp. 722-734, 2019.

[4] M. M. Salah, A. A. Ashour, T. M. Abdelghany et al., "Pirfenidone alleviates concanavalin A-induced liver fibrosis in mice," Life Sciences, vol. 239, Article ID 116982, 2019.

[5] C. Fan, F. R. Wu, J. F. Zhang et al., "A network pharmacology approach to explore the mechanisms of shugan jianpi formula in liver fibrosis," Evidence-Based Complementary and Alternative Medicine, vol. 2020, Article ID 4780383, 13 pages, 2020.

[6] J. Y. Wu, S. K. Hong, X. K. Xie et al., "A network pharmacology-based study on the anti-lung cancer effect of dipsaci radix," Evidence-Based Complementary and Alternative Medicine, vol. 2020, Article ID 7424061, 9 pages, 2020.

[7] C. Zhang, K. Zhang, X. Tian et al., "Hepatoprotective effect of hugan buzure keli on carbon tetrachloride induced-hepatic fibrosis in mice," Journal of Shihezi University, vol. 34, no. 4, pp. 355-359, 2016.

[8] X. J. Yang, J. H. Yang, J. P. Hu et al., "Study on the effect of uygur presciption hugan buzure on acute liver injury of rats," Journal of Xinjiang Medical University, vol. 40, no. 11, pp. 1479-1483, 2017.

[9] J. Ru, P. Li, J. Wang et al., "TCMSP: a database of systems pharmacology for drug discovery from herbal medicines," Journal of Cheminformatics, vol. 6, no. 13, 2014.

[10] M. Brüstle, B. Beck, T. Schindler, W. King, T. Mitchell, and T. Clark, "Descriptors, physical properties, and drug-likeness," Journal of Medicinal Chemistry, vol. 45, no. 16, pp. 3345-3355, 2002.

[11] Z. Liu, F. Gou, Y. Wang et al., "BATMAN-TCM.: a bioinformatics analysis tool for molecular mechanism of traditional Chinese medicine," Scientific Reports, vol. 6, p. 21146, 2016.

[12] A. Daina, O. Michielin, and V. Zoete, "SwissTargetPrediction: updated data and new features for efficient prediction of protein targets of small molecules," Nucleic Acids Research, vol. 47, no. W1, pp. W357-W364, 2019.

[13] G. Stelzer, N. Rosen, I. Plaschkes et al., "The geneCards suite: from gene data mining to disease genome sequence analyses," Current Protocol in Bioinformatics, vol. 54, no. 1, pp. 1303113033, 2016.

[14] D. Szklarczyk, A. L. Gable, D. Lyon et al., "STRING v11: protein-protein association networks with increased coverage, supporting functional discovery in genome-wide experimental datasets," Nucleic Acids Research, vol. 47, no. D1, pp. D607-D613, 2019.

[15] G. Dennis, B. T. Sherman, D. A. Hosack et al., "DAVID.: database for annotation, visualization, and integrated discovery," Genome Biology, vol. 4, no. 9, pp. 418-427, 2003.

[16] M. Ashburner, C. A. Ball, J. A. Blake et al., "Gene ontology: tool for the unification of biology," Nature Genetics, vol. 25, no. 1, pp. 25-29, 2000. 
[17] M. Qiao, J. H. Yang, Y. Zhao et al., "Antiliver fibrosis screening of active ingredients from Apium graveolens L. seeds via GC-TOF-MS and UHPLC-MS/MS," EvidenceBased Complementary and Alternative Medicine, vol. 2020, Article ID 8321732, 12 pages, 2020.

[18] I. H. Kim, J. Xu, X. Liu et al., "Aging increases the susceptibility of hepatic inflammation, liver fibrosis and aging in response to high-fat diet in mice," Journal of the American Aging Association, vol. 38, no. 4, 12 pages, 2016.

[19] A. Chamorro-Jorganes, M. Y. Lee, E. Araldi et al., "VEGFinduced expression of miR-17-92 cluster in endothelial cells is mediated by ERK/ELK1 activation and regulates angiogenesis," Circulation Research, vol. 118, no. 1, pp. 38-47, 2016.

[20] Y. Zhang, S. Li, S. Huang et al., "IL33/ST2 contributes to airway remodeling via $\mathrm{p}$-JNK MAPK/STAT3 signaling pathway in OVA-induced allergic airway inflammation in mice," Experimental Lung Research, vol. 45, no. 57, 11 pages, 2019.

[21] S. Kothakota, T. Azuma, C. Reinhard et al., "Caspase-3-generated fragment of gelsolin: effector of morphological change in apoptosis," Science, vol. 278, no. 5336, pp. 294-298, 1997.

[22] P. Melgar, M. Perramon, W. Jimenez et al., "Roles of the hepatic endocannabinoid and apelin systems in the pathogenesis of liver fibrosis," Cells, vol. 8, no. 11, pp. 265-276, 2019.

[23] Y. G. Tao, X. F. Huang, J. Y. Wang et al., "Exploring molecular mechanism of huangqi in treating heart failure using network pharmacology," Evidence-Based Complementary and Alternative Medicine, vol. 2020, Article ID 6473745, 17 pages, 2020.

[24] M. Qiao, J. H. Yang, Y. Zhu et al., "Transcriptomics and proteomics analysis of system-level mechanisms in the liver of apigenin-treated fbrotic rats," Life Sciences, vol. 248, Article ID 117475, 2020.

[25] G. Zhu, X. Liu, H. Li et al., "Kaempferol inhibits proliferation, migration, and invasion of liver cancer HepG2 cells by downregulation of microRNA-21," International Journal of Immunopathology and Pharmacology, vol. 32, 2018.

[26] G. Gyeong, K. Yeon, A. Hyun et al., "Antifibrotic effect of smad decoy oligodeoxynucleotide in $\mathrm{CCl}_{4}$-induced hepatic fibrosis animal model," Molecules, vol. 23, no. 1991, 13 pages, 2019.

[27] B. G. Fernández, D. Sánchez, I. Crespo et al., "Melatonin attenuates dysregulation of the circadian clock pathway in mice with $\mathrm{CCl}_{4}$-induced fibrosis and human hepatic stellate cells," Frontier in Pharmacology, vol. 9, no. 556, 11 pages, 2018.

[28] Y.-N. Zhou, M.-Y. Sun, Y.-P. Mu et al., "Xuefuzhuyu decoction inhibition of angiogenesis attenuates liver fibrosis induced by CCl4 in mice," Journal of Ethnopharmacology, vol. 153, no. 3, pp. 659-666, 2014.

[29] Z. A. Ghoreshi, R. Kabirifar, A. Khodarahmi et al., "The preventive effect of atorvastatin on liver fibrosis in the bile duct ligation rats via antioxidant activity and down-regulation of Rac1 and NOX1," Iranian Journal of Basic Medical Science, vol. 23, no. 1, pp. 30-35, 2020.

[30] Z. Yan, K. Qu, J. Zhang et al., "CD147 promotes liver fibrosis progression via VEGF-A/VEGFR2 signalling-mediated crosstalk between hepatocytes and sinusoidal endothelial cells," Clinical Science, vol. 129, no. 8, pp. 699-710, 2015.

[31] P. M. Waziri, R. Abdullah, R. Rosli et al., "Clausenidin induces caspase 8-dependent apoptosis and suppresses production of VEGF in liver cancer cells," Asian Pacific Journal of Cancer Prevention, vol. 19, no. 4, pp. 917-922, 2017.
[32] S. H. Lee, Y. J. Lee, C. H. Song, Y. K. Ahn, and H. J. Han, "Role of FAK phosphorylation in hypoxia-induced hMSCS migration: involvement of VEGF as well as MAPKS and eNOS pathways," American Journal of Physiology-Cell Physiology, vol. 298, no. 4, pp. C847-C856, 2010.

[33] L. Zuo, Y. Zhu, L. Hu et al., "PI3-kinase/Akt pathway-regulated membrane transportation of acid-sensing ion channel 1a/ calcium ion influx/endoplasmic reticulum stress activation on PDGF induced HSC activation," Journal of Cellular and Molecular Medicine, vol. 6, no. 23, pp. 3940-3950, 2019.

[34] A. Xu, Y. Li, W. Zhao et al., "PHP14 regulates hepatic stellate cells migration in liver fibrosis via mediating TGF- $\beta 1$ signaling to PI3K $\gamma / \mathrm{AKT} / \mathrm{Rac1}$ pathway," Journal of Molecular Medicine, vol. 96, no. 2, pp. 119-133, 2018.

[35] W. Zhao, J. Ye, and Z.-J. Wang, "Embryonic liver fordin is involved in glucose glycolysis of hepatic stellate cell by regulating PI3K/Akt signaling," World Journal of Gastroenterology, vol. 22, no. 38, pp. 8519-8527, 2016.

[36] Y. Wang, Y. Huang, F. Guan et al., "Hypoxia-inducible factor1alpha and MAPK co-regulate activation of hepatic stellate cells upon hypoxia stimulation," PLoS One, vol. 8, no. 9, Article ID e74051, 2012.

[37] H. Wu, X. W. Cheng, L. Hu et al., "Cathepsin S activity controls injury-related vascular repair in mice via the TLR2mediated p38MAPK and PI3K-Akt/p-HDAC6 signaling pathway," Arteriosclerosis, Thrombosis, and Vascular Biology, vol. 36, no. 8, pp. 1549-1557, 2016.

[38] M. G. Elfeky, E. M. Mantawy, A. M. Gad et al., "Mechanistic aspects of antifibrotic effects of honokiol in Con A-induced liver fibrosis in rats: emphasis on TGF- $\beta /$ SMAD/MAPK signaling pathways," Life Sciences, vol. 240, Article ID 117096, 2019.

[39] Z.-b. Zhao, K. Ji, X.-y. Shen et al., "Di (2-ethylhexyl) phthalate promotes hepatic fibrosis by regulation of oxidative stress and inflammation responses in rats," Environmental Toxicology and Pharmacology, vol. 68, no. 5, pp. 109-119, 2019.

[40] N. Liu, J. Feng, X. Lu et al., "Isorhamnetin inhibits liver fibrosis by reducing autophagy and inhibiting extracellular matrix formation via the TGF- $\beta 1 / \mathrm{smad} 3$ and TGF- $\beta 1 / \mathrm{p} 38$ MAPK pathways," Mediators of Inflammation, vol. 2019, no. 5, pp. 1-14, Article ID 6175091, 2019.

[41] X.-w. Gong, Y.-j. Xu, Q.-h. Yang et al., "Effect of soothing gan (liver) and invigorating pi (spleen) recipes on TLR4-p38 MAPK pathway in kupffer cells of non-alcoholic steatohepatitis rats," Chinese Journal of Integrative Medicine, vol. 25, no. 3, pp. 216-224, 2019.

[42] B. Nath and G. Szabo, "Hypoxia and hypoxia inducible factors: diverse roles in liver diseases," Hepatology, vol. 55, no. 2, pp. 622-633, 2012.

[43] J. Deng, Q. Huang, Y. Wang et al., "Hypoxia-inducible factor1alpha regulates autophagy to activate hepatic stellate cells," Biochemical and Biophysical Research Communications, vol. 454, no. 2, pp. 328-334, 2014.

[44] H. Sun, G. Chen, B. Wen et al., "Oligo-peptide I-C-F-6 inhibits hepatic stellate cell activation and ameliorates $\mathrm{CCl}_{4}$ -induced liver fibrosis by suppressing NF- $\kappa \mathrm{B}$ signaling and Wnt/ $\beta$-catenin signaling," Journal of Pharmacological Sciences, vol. 136, no. 3, pp. 133-141, 2018.

[45] B. Wu, R. Wang, S. Li et al., "Antifibrotic effects of Fraxetin on carbon tetrachloride-induced liver fibrosis by targeting NF$\kappa \mathrm{B} / \mathrm{I} \kappa \mathrm{B} \alpha$, MAPKs and Bcl-2/bax pathways," Pharmacological Reports, vol. 71, no. 3, pp. 409-416, 2019.

[46] Z. Wei, D. Zhao, Y. Zhang et al., "Rosiglitazone ameliorates bile duct ligation-induced liver fibrosis by down-regulating 
NF- $\kappa$ B-TNF- $\alpha$ signaling pathway in a $\operatorname{PPAR} \gamma$-dependent manner," Biochemical and Biophysical Research Communications, vol. 519, no. 4, pp. 854-860, 2019.

[47] E. Borkham, C. R. Roeyen, T. Ostendorf et al., "Pro-fibrogenic potential of PDGF-D in liver fibrosis," Journal of Hepatology, vol. 46, no. 6, pp. 1064-1074, 2007.

[48] J. Iruolagbe, R. Ohanador, B. Amene-Imananagha, A. Abubakar, O. Jessica, and A. W. Obianemmie, "Prevalence of liver-fibrosis using FIB-4 in HIV-patients on anti-retroviral therapy in Nigeria and CD4 and TNF as predictors," Asian Journal of Research in Medical and Pharmaceutical Sciences, vol. 6, no. 4, pp. 1-9, 2019.

[49] Z. Deng, S. Zhang, S. Ge, F. Kong, S. Cao, and Z. Pan, "Gexiazhuyu Decoction attenuates carbon tetrachloride-induced liver fibrosis in mice partly via liver angiogenesis mediated by myeloid cells," Medical Science Monitor, vol. 25, no. 6, pp. 2835-2844, 2019.

[50] M. Khalili, F. G. Firuozsalari, A. Naeimabadi et al., "Curcumin modulates the level of IL-17 and IL-10 cytokines in two models of experimental liver injury in male rats," Journal of Kerman University of Medical Sciences, vol. 25, no. 2, pp. 104-117, 2018. 